\title{
SYNTHESIS AND ANTIBACTERIAL ACTIVITY OF SOME NOVEL EPOXYCHALCONES DERIVED FROM CHALCONES
}

\author{
S.L. Kumbhare \\ Shri Shivaji Science \& Arts College, Chikhli Dist.- Buldana \\ Email - infoslkumbhare@yahoo.com
}

\begin{abstract}
-
Novel Epoxychalcones have been synthesized by reacting newly synthesized chalcones with Hydrogen peroxide. These Epoxychalcones have been confirmed by IR and NMR spectral analysis. These Epoxychalcones were screened for antibacterial activity.

Keywords - Chalcones, Epoxychalcones, Antibacterial activity.

\section{Introduction -}

Chalcones and its epoxide derivatives (Epoxychalcones) are reported to have antibacterial, antifungal, antiparasitic, antiprotozoal, antimitotic, antitubercular and insect repellent activity. Epoxide derivatives of chalcones are very important precursor in the synthesis of many natural products and drug molecules. Great attention has been dedicated on the development of methodologies allowing the epoxidation of chalcones. Keeping in view These observations, it was planned to synthesize some novel Epoxychalcones bu reacting with Hydrogen peroxide. Epoxychalcones have been confirmed by Ir and 1H NMR spectal analysis. The Epoxychalcones were tested for antibacterial activity by agar cup plate method against the Strains of S. aureus and E. coli.
\end{abstract}

Melting point were recorded in liquid paraffin bath using open end capillaries and are uncorrected. The IR spectra were run on Shimadzu FTIR spectrophotometer in $\mathrm{KBr}$ pellets. $1 \mathrm{H}$ NMR spectra were recorded on a Bruker $300 \mathrm{MHz}$ NMR Spectrometer in CDCl3/DMSO-d6 using TMS as internal reference. Thin layer Chromatography was performed on silica gel (Merck). 


\section{Method -}

Chalcones were synthesized and then epoxidated with hydrogen peroxide as per following method.

\subsection{Synthesis of novel chacones(I) -}

To a solution of P-bromo acetophenone $(0.01 \mathrm{~mol})$ in oxygen free distilled ethanol, different aromatic aldehydes such as O-Chlorobenzaldehyde, PChlorobenzaldehyde, P-Hydroxy benzaldehyde, PMethoxybenzaldehyde(anisaldehyde), Salicylaldehyde, P-dimethyl aminobenzaldehyde, Furfural, Vaniline(4-Hydroxy,3-methoxy benzaldehyde) $(0.01 \mathrm{~mol})$ and $5 \mathrm{ml}$ of $40 \%$ potassium Hydroxide were added. The reaction mixture was refluxed for $4 \mathrm{~h}$. It was cooled to room temperature and poured into ice-cold water. A solid mass so obtained was filtered, washed with sodium bicarbonate solution followed by water and then dried. It was crystallized from acetone to give pure crystals of Chalcones(I).

\subsection{Synthesis of Epoxychalcones(II) -}

To a cooled solution of chalcones(I)(1.0g) in acetone (20ml) was added in aqueous solution of sodium hydroide $(1.2 \mathrm{ml}, 8 \%)$. The reaction mixture was then cooled in ice-bath and Hydrogen peroxide $(2 \mathrm{ml}, 30 \%)$ was added. The contents were shaken for $1 \mathrm{~h}$ with cooling at 15 minute interval and left overnight. The solid mass, which separated out, was filtered and dried to give tlc.

\section{Result and discussion -}

All the chalcones(I) were obtained to good yields in the range of $60-70 \%$ and gave red colour with Conc. Sulphuric acid. All the chalcones were analysed by Melting point, IR and NMR spectra. Similarly all synthesized Epoxychalcones were obtained in moderate yield in the range of 42-50\%. All Epoxychalcones were characterized by Melting point, IR and NMR Spectra. All the 
Epoxychalcones were tested for antibacterial activity by agar cup -plate method against the Strains of S.aureus and E.coli.

\section{Conclusion-}

The antibacterial activity of all Epoxychalcones were found to be active against test organisms. Compound containing bromo group at C-4 position of the phenyl ring exhibited significant activity while other showed moderate activity against the two tested microorganisms.

\section{References}

A.G. Doshi and B.J. Ghiya, (1979). Indian J. Chem., 17B,509.

J.K. Makrandi and S.K. Grover, (1982). Indian J. Chem., 21B,543.

W.B. Geirger and J.E. Conn, (1995). J. Am. Chem. Soc.,67,112.

S.V.Krishna, A. rajshekhar, T.K.Reddy and M.S. Naidu, (1988). Curr.Sci. (India), 57, 129 .

V.V.Laxmi, P.Shridhar and H.Polasa, (1985). Indian J. Pharma. Sci.,47,202. 